\title{
Real-time Dynamic Coupling of GPC-enhanced Diffraction-limited Focal Spots.
}

Villangca, Mark Jayson; Bañas, Andrew Rafael; Kopylov, Oleksii; Palima, Darwin; Glückstad, Jesper

\section{Published in:}

Proceedings of SPIE

Link to article, DOI:

$10.1117 / 12.2079148$

Publication date:

2015

Document Version

Publisher's PDF, also known as Version of record

Link back to DTU Orbit

Citation (APA):

Villangca, M. J., Bañas, A. R., Kopylov, O., Palima, D., \& Glückstad, J. (2015). Real-time Dynamic Coupling of GPC-enhanced Diffraction-limited Focal Spots. In Proceedings of SPIE (Vol. 9379). [93790M] SPIE -

International Society for Optical Engineering. Proceedings of SPIE - The International Society for Optical Engineering https://doi.org/10.1117/12.2079148

\section{General rights}

Copyright and moral rights for the publications made accessible in the public portal are retained by the authors and/or other copyright owners and it is a condition of accessing publications that users recognise and abide by the legal requirements associated with these rights.

- Users may download and print one copy of any publication from the public portal for the purpose of private study or research.

- You may not further distribute the material or use it for any profit-making activity or commercial gain

- You may freely distribute the URL identifying the publication in the public portal 


\title{
Real-time Dynamic Coupling of GPC-enhanced Diffraction-limited Focal Spots
}

\author{
Mark Villangca*, Andrew Bañas, Oleksii Kopylov, Darwin Palima and Jesper Glückstad \\ DTU Fotonik, Dept. of Photonics Engineering, Technical University of Denmark \\ DK-2800 Kgs. Lyngby, Denmark \\ *Email: majv@fotonik.dtu.dk \\ www.ppo.dk
}

\begin{abstract}
We have previously demonstrated on-demand dynamic coupling of an optically manipulated wave-guided optical waveguide (WOW) using diffractive techniques on a "point and shoot" approach. In this work, the generation of the coupling focal spots is done in real-time following the position of the WOW. Object-tracking routine has been added in the trapping program to get the position of the WOW. This approach allows continuous coupling of light through the WOWs which may be useful in some application. In addition, we include a GPC light shaper module in the holography setup to efficiently illuminate the spatial light modulator (SLM). The ability to switch from on-demand to continuous addressing with efficient illumination leverages our WOWs for potential applications in stimulation and nonlinear optics.
\end{abstract}

Keywords: Microfabrication, optical trapping, object tracking, Generalized Phase Contrast

\section{INTRODUCTION}

Optical trapping has progressed from single tightly focused beam to orchestrated movement of multiple traps moving multiple objects ${ }^{1,2}$. The trapped object is no longer restricted to polystyrene bead but also extended objects that are fabricated using two-photon fabrication $(2 \mathrm{PP})^{3}$. The use of $2 \mathrm{PP}$ allows flexibility in the design of the structure so that it can perform various tasks. One example is the optically-actuated surface scanning probe to investigate surface topography ${ }^{4}$. Aside from tightly focused traps, counterpropagating (CP) beams can also be used in trapping. We have demonstrated this with beads ${ }^{5}$ and with fabricated extended objects that is used for microassembly ${ }^{6,7}$. One advantage of using CP beams is that one can use low NA objective lenses to relay the trapping beams in the sample. The large working distance of low NA objectives allows the possibility of adding a side view imaging of the sample as what we did in our Biophotonics Workstation (BWS $)^{8}$. In our recent work, we fabricated free-floating waveguides that can be optically manipulated coined as wave-guided optical waveguide $(\mathrm{WOW})^{9}$. The WOW can serve as a structure-mediated tool for redirecting light ${ }^{10}$. To allow dynamic coupling of light through the waveguide, we added holography setup that modulates the coupling beam in our BWS ${ }^{11}$.

In this work, further improvement has been made by adding object tracking routine that will allow real-time coupling of the WOW. This will leverage the capability of the WOWs for potential applications in photostimulation and nonlinear optics.

\section{METHODS}

The WOWs are manipulated using the spherical handles held by CP beams $(\lambda=1070 \mathrm{~nm})$. Lateral movement of the WOWs is accomplished by simply dragging the CP beams along the lateral direction while the axial movement is done by changing the intensity ratio of the CP beams. As the WOW is moved in 3D space, we require the coupling beam $(\lambda=532 \mathrm{~nm})$ to follow it for continuous addressing. The lateral displacements $\Delta x^{\prime}$ and $\Delta y^{\prime}$ of the WOW can be readily obtained from the trapping interface since the CP beams uses an imaging geometry and thus simple scaling is needed for the hologram calculation. Since we have no direct access in the axial coordinate due to the nature of the trapping beams, we use object tracking routine on the side imaging to automatically get the axial displacement $\Delta z^{\prime}$. A fluorescence dye is added to the trapping medium to aid in visualizing the coupling beam using the side imaging. The procedure is summarized in figure 1 . 


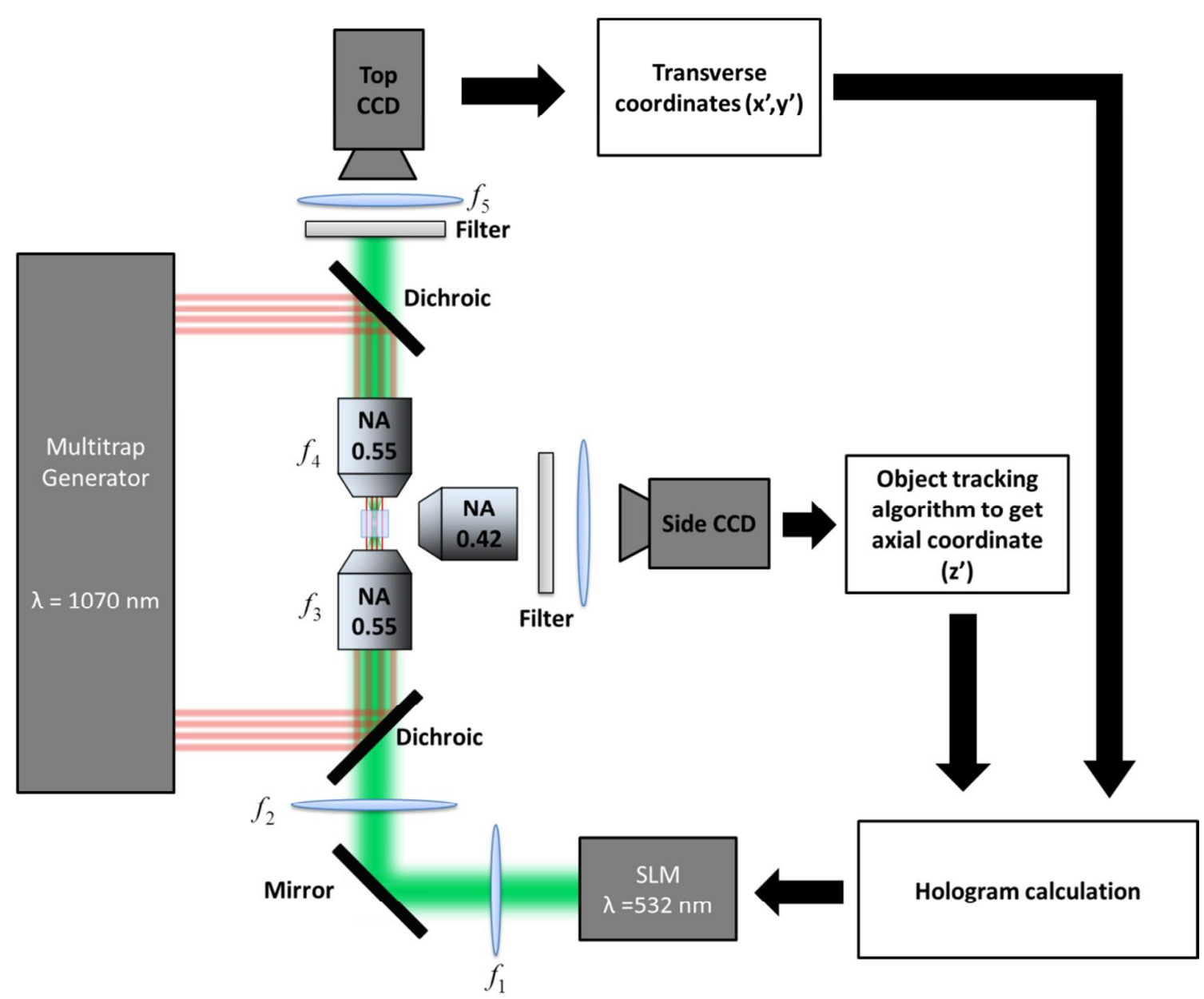

Figure 1. Schematic diagram of real-time coupling of a WOW. The coordinates of the WOW is obtained automatically for the calculation of the appropriate hologram.

The required holograms for the lateral and axial movement of the coupling beam are calculated using the blaze grating and quadratic phases respectively and are given by

$$
\begin{gathered}
\phi_{\text {lateral }}(x, y)=\frac{2 \pi}{\lambda f}\left(x \Delta x^{\prime}+y \Delta y^{\prime}\right) \\
\phi_{\text {axial }}(x, y)=-\frac{\pi \Delta z^{\prime}}{\lambda f}\left(x^{2}+y^{2}\right)
\end{gathered}
$$

where $f$ is the focal length of the Fourier transforming lens. In some cases, an offset is necessary to have a convenient coordinate for both the trapping beam and coupling beam. Thus the effective phase for coupling beam is given by

$$
\phi_{\text {eff }}(x, y)=\bmod \left(\phi_{\text {offset }}+\phi_{\text {lateral }}+\phi_{\text {axial }}, 2 \pi\right)
$$

The holographic addressing of the WOW can be visualized as shown in figure 2 . 


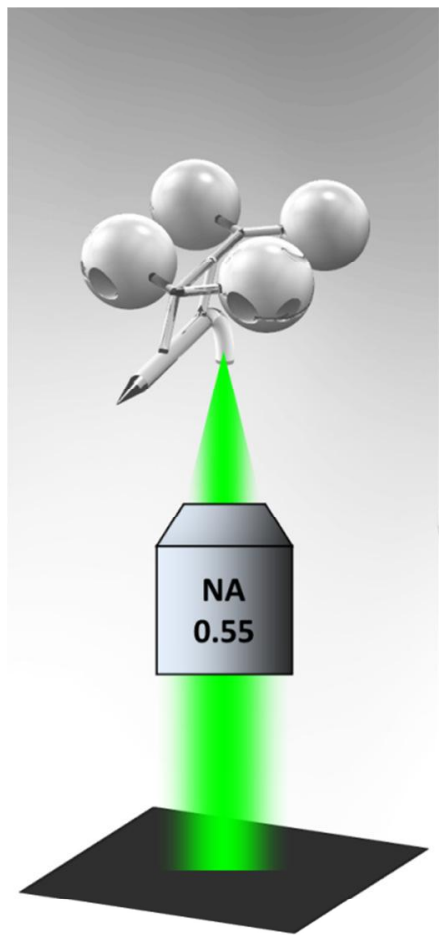

unmodulated

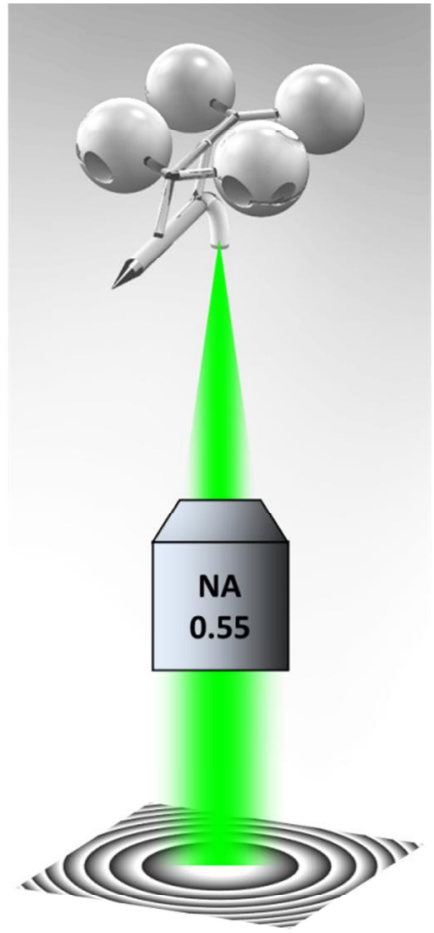

lens phase

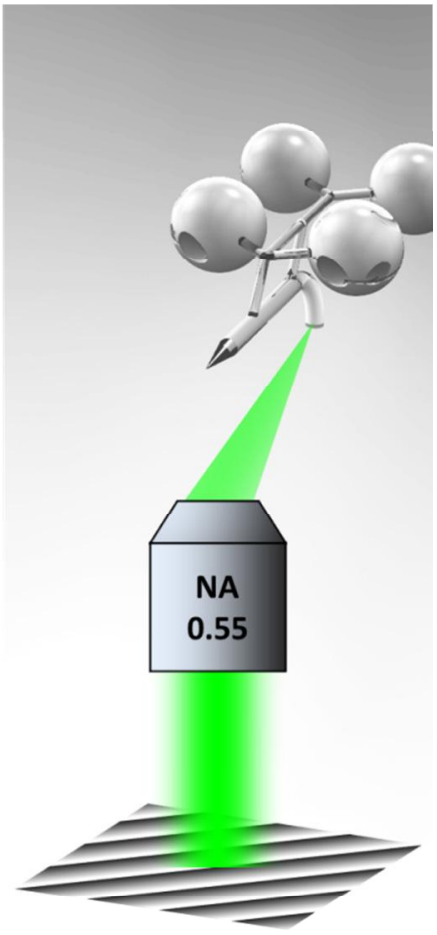

blazed grating phase

Figure 2. Graphical demonstration of holographic addressing of the WOW. The WOW is moved in 3D space using the counterpropagating beams holding the spherical handles. The holograms are used to modulate the coupling beam to dynamically follow the position of the WOW in real-time.

To increase the efficiency of the coupling beam, a preliminary beam shaping using the Generalized Phase Contrast method $^{12}$ is done to match the shape of the SLM. A compact module containing the main components of GPC is added in the holography setup as shown in figure 3. We call this module as the GPC light shaper (LS). The size of the phase mask and phase contrast filter (PCF) is optimized according to the values in literature ${ }^{13}$. The use of GPC LS allows efficient photon management compared to the more common practice of amplitude masking ${ }^{13,14}$.

The GPC LS works by introducing a phase shift to the incident Gaussian beam using a phase mask that has a phase shifting region corresponding to the shape of the SLM (Hamamatsu Photonics, $798 \times 600$ pixels, $9.9 \mathrm{~mm} \times 7.5 \mathrm{~mm}$ active area). A lens takes the Fourier transform of the phase shifted field in the plane of the PCF which creates a synthetic reference wave from the low spatial frequencies. A second lens takes another Fourier transform of the field and the resulting pattern in the output plane is a contiguous intensity distribution corresponding to the phase mask. Unlike the common method of hard-truncation where photons are discarded, the GPC LS redirects photons to where they are needed. 


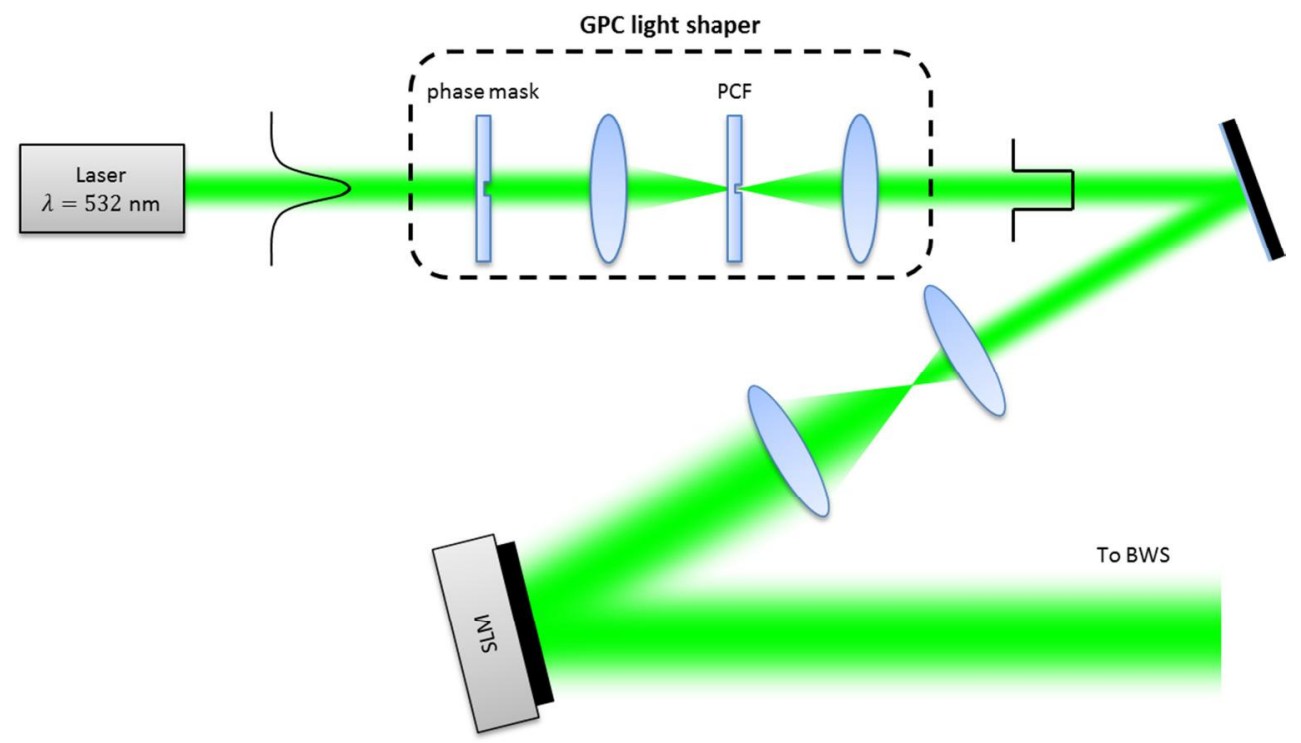

Figure 3. Schematic diagram of the holography setup. The GPC LS is placed before the SLM to perform preliminary beam shaping to match the shape of the SLM. Unlike hard-truncation where many of the photons are discarded, the GPC LS redirects the photons to where it is needed.

\section{RESULTS}

Real-time coupling has been tested for lateral and axial displacement of the WOW separately. Figures 4 and 5 show snapshots from experiments performing real-time coupling during WOW motion. Lateral movement of WOW is done by dragging the trap using a computer interface. The coordinate variables are then grabbed to calculate the required grating phase. For axial addressing, as shown in figure $2 \mathrm{~b}$, the position of the WOW is obtained from the built-in object tracking routine in LabVIEW. The obtained axial displacement is then used to calculate the required quadratic phase. The axial shifting of the coupling spots can be seen clearly from the fluorescence of the trapping medium. The use of GPC light shaper prior to phase modulation using a SLM produces more intense coupling spot which is desirable for addressing multiple WOWs.
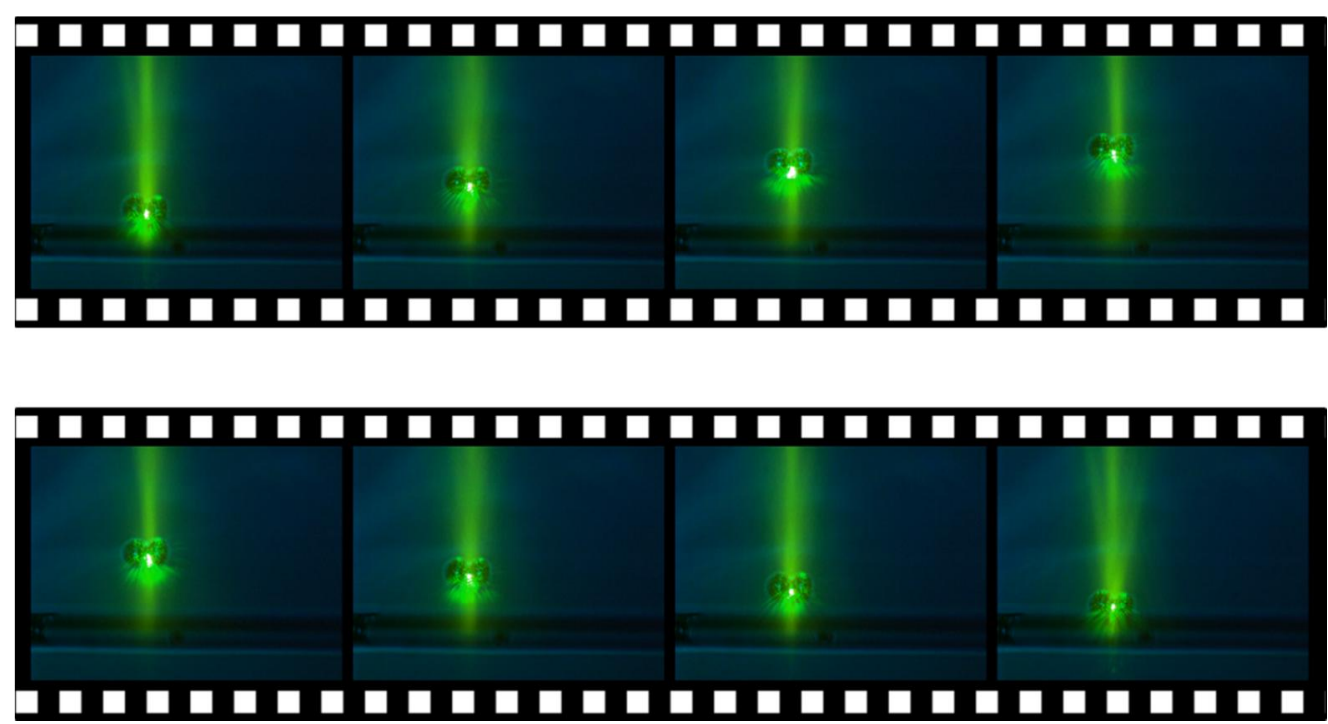

Figure 4. Snapshots of experimental result demonstrating real-time coupling along the axial direction (see Vided). http://dx.doi.org/10.1117/12.2079148.1 

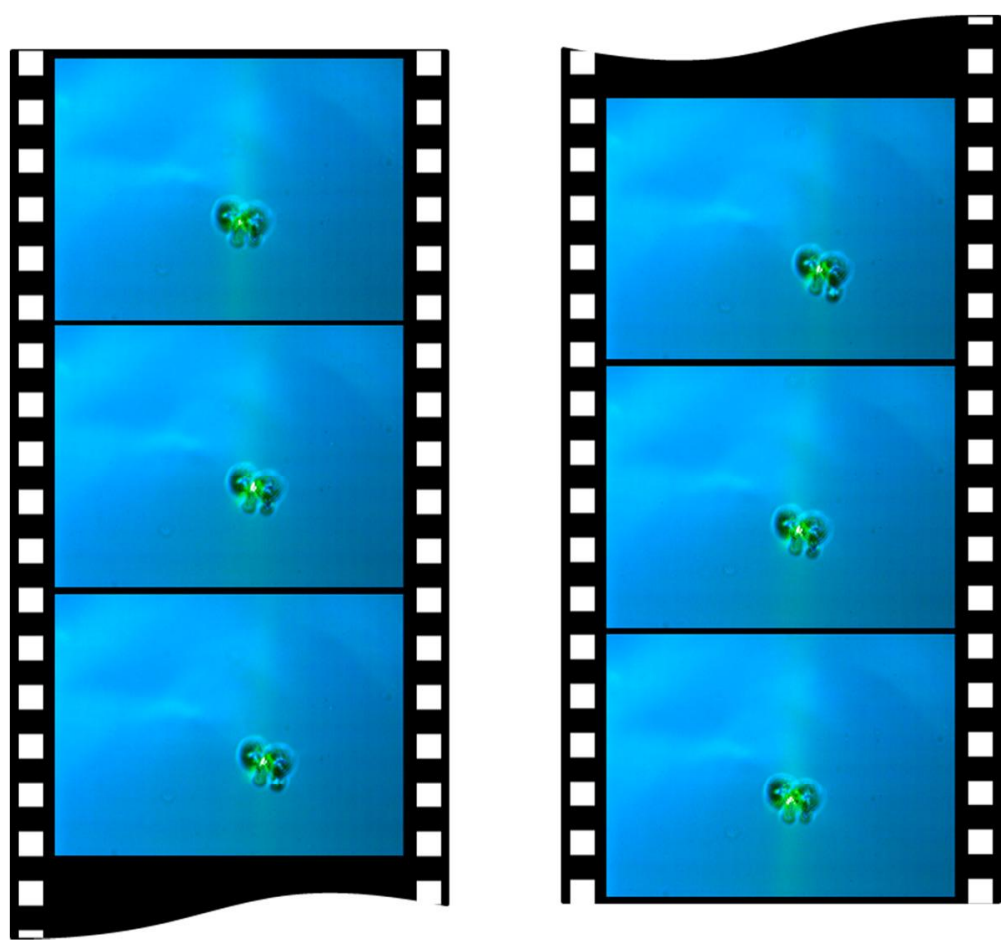

Figure 5. Snapshots of experimental result demonstrating real-time coupling along the lateral direction (see Videฉ). http://dx.doi.org/10.1117/12.2079148.2

\section{CONCLUSION}

We have shown real-time coupling of the wave-guided optical waveguides by using object tracking algorithm. The ability to switch between on-demand and continuous coupling offers versatility in the WOWs for potential applications in photostimulation and nonlinear optics. The addition of GPC light shaper in the holography setup allows efficient formation of high intensity light spots that is suitable when addressing multiple WOWs.

\section{ACKNOWLEDGMENTS}

This work is supported by Enhanced Spatial Light Control in Advanced Optical Fibres (e-space), a project financed by Innovation Fund Denmark and the Copenhagen Cleantech Cluster (CCC) for the GAP funding. We also acknowledge the support from Hamamatsu Photonics.

\section{REFERENCES}

[1] Ashkin, A., “Acceleration and Trapping of Particles by Radiation Pressure,” Phys. Rev. Lett. 24(4), 24-27 (1970).

[2] Curtis, J. E., Koss, B. A., Grier, D. G., “Dynamic holographic optical tweezers,” Opt. Commun. 207, 169-175 (2002).

[3] Palima, D., Glückstad, J., "Gearing up for optical microrobotics: micromanipulation and actuation of synthetic microstructures by optical forces," Laser Photon. Rev. 7(4), 478-494 (2013).

[4] Phillips, D. B., Gibson, G. M., Bowman, R., Padgett, M. J., Hanna, S., Carberry, D. M., Miles, M. J., Simpson, S. H., “An optically actuated surface scanning probe," Opt. Express 20(28), 29679-29693 (2012).

[5] Rodrigo, P. J., Perch-Nielsen, I. R., Alonzo, C. A., Glückstad, J., "GPC-based optical micromanipulation in 3D real-time using a single spatial light modulator," Opt. Express 14(26), 13107-13112 (2006). 
[6] Rodrigo, P. J., Gammelgaard, L., Bøggild, P., Perch-Nielsen, I., Glückstad, J., “Actuation of microfabricated tools using multiple GPC-based counterpropagating-beam traps," Opt. Express 13(18), 6899-6904 (2005).

[7] Rodrigo, P. J., Kelemen, L., Palima, D., Alonzo, C. A., Ormos, P., Glückstad, J., "Optical microassembly platform for constructing reconfigurable microenvironments for biomedical studies," Opt. Express 17(8), 65786583 (2009).

[8] Ulriksen, H.-U., Thøgersen, J., Keiding, S., Perch-Nielsen, I. R., Dam, J. S., Palima, D. Z., Stapelfeldt, H., Glückstad, J., "Independent trapping, manipulation and characterization by an all-optical biophotonics workstation," J. Eur. Opt. Soc. Rapid Publ. 3, 08034 (2008).

[9] Palima, D., Bañas, A., Vizsnyiczai, G., Kelemen, L., Ormos, P., Glückstad, J., "Wave-guided optical waveguides," Opt. Express 20(3), 2004-2014 (2012).

[10] Glückstad, J., Bañas, A., Aabo, T., Palima, D., "Structure-mediated micro-to-nano coupling using sculpted light and matter," Proc. SPIE Vol. 8424 8424, D. L. Andrews, J.-M. Nunzi, and A. Ostendorf, Eds., 84241L (2012).

[11] Villangca, M., Bañas, A., Palima, D., Glückstad, J., "Dynamic diffraction-limited light-coupling of 3Dmaneuvered wave-guided optical waveguides," Opt. Express 22(15), 17880-17889 (2014).

[12] Glückstad, J., Palima, D., [Generalized Phase Contrast: Applications in Optics and Photonics], Springer Series in Optical Sciences (2009).

[13] Bañas, A., Palima, D., Villangca, M., Aabo, T., Glückstad, J., "GPC light shaper for speckle-free one-and twophoton contiguous pattern excitation," Opt. Express 22(5), 5299-5310 (2014).

[14] Bañas, A., Kopylov, O., Villangca, M., Palima, D., Glückstad, J., "GPC light shaper: static and dynamic experimental demonstrations," Opt. Express 22(20), 23759-23769 (2014). 